\title{
Instrumentos para la participación ciudadana y requisitos para su efectividad
}

\author{
Joan Font Fàbregas \\ José Luis Fernández-Martínez \\ Patricia García Espín
}

\begin{abstract}
Sumario: I. Introducción.-II. ¿Cómo podríamos evaluar la efectividad de procesos e instrumentos participativos?.-III. Procesos participativos y políticas públicas. - IV. Cambios en el contexto político local. -V. Conclusiones. -VI. Referencias
\end{abstract}

\section{Introducción}

¿Es efectivo nuestro coche? ¿Lo es el funcionamiento de un hospital o de una escuela pública? ¿Es efectivo un seleccionador nacional de balonmano? Sin duda, se trata de tres realidades muy distintas que no se pueden juzgar desde un mismo parámetro. Sin embargo, cuando los comparamos a un proceso participativo, el coche, el hospital o el seleccionador tienen algo en común: resulta relativamente sencillo decir cuáles son los objetivos básicos desde los que se justifica su existencia.

Aunque en realidad el coche pueda servir para ensalzar el prestigio de su conductor o el hospital para hacer pasar a la historia local a determinado dirigente político, los principales objetivos confesables de cada uno de ellos son más obvios: hay que transportar y hacer kilómetros con comodidad, curar enfermos y salvar vidas o ganar copas de Europa. Pero no podemos aplicar esa misma lógica de forma automática a un proceso participativo porque para saber cómo medimos la efectividad necesitaríamos la respuesta a una pregunta que muy a menudo no sabemos responder ¿Para qué queremos los instrumentos participativos? ¿Qué esperamos de ellos? ¿Qué objetivos concretos esperamos alcanzar?

A pesar de la dificultad de saber si un proceso participativo es efectivo partiendo de esta ambigüedad, en las próximas páginas trataremos de acercarnos a este tema. Para ello, procederemos en tres pasos. En el primer apartado pondremos encima de la mesa algunos de los posibles criterios para valorar la efectividad de un proceso participativo. Como vere- 
mos, algunos de estos criterios han recibido mayor atención empírica que otros. Por ello, en este artículo nos centraremos en uno de los menos estudiados, y a su vez relacionado con los resultados más tangibles de los procesos participativos. A su vez, dividiremos estos posibles resultados en dos grandes grupos. En primer lugar, en el apartado tres, abordaremos uno de los más evidentes, las políticas públicas a las que pueden dar lugar las resoluciones adoptadas en estos procesos. Así, en ese apartado veremos los resultados de un proyecto de investigación reciente en el que hemos analizado hasta qué punto las propuestas surgidas de los procesos participativos se convierten o no en políticas y cuáles son los factores que influyen en que una propuesta tenga más o menos posibilidades de ser implementada. Por su parte, en el apartado cuatro nos moveremos hacia un tipo de resultados más culturales, aquellos que tienen que ver con revitalizar la sociedad civil de la comunidad y/o mejorar las relaciones entre esta y la administración pública. En este apartado presentaremos también resultados del mismo proyecto, apuntando cuáles son algunos de los efectos más claros que hemos detectado en este ámbito y poniendo ejemplos de casos específicos donde se han producido. Por último, cerramos el artículo con unas breves conclusiones donde retomamos el debate sobre qué podemos esperar de los procesos participativos.

Aunque la gran mayoría de las ideas que discutiremos son probablemente aplicables a procesos en distintos niveles y administraciones, la base empírica de la que partimos proviene fundamentalmente de experiencias participativas locales desarrolladas en Andalucía, Cataluña y Madrid. A través de estas experiencias podemos extraer algunas enseñanzas para su aplicación a realidades que, presentando similitudes y diferencias con las mismas, pueden resultar útiles para el contexto vasco.

\section{II. ¿Cómo podríamos evaluar la efectividad de procesos e instrumentos participativos?}

Se ha dicho en muchas ocasiones que el instrumento participativo central en las democracias representativas, los procesos electorales, tienen tres funciones principales: renovar la legitimidad democrática que sustenta el funcionamiento de la vida política, dar lugar a una representación de los intereses de los distintos ciudadanos y grupos sociales, y permitir la formación de un gobierno (Carreras y Vallés, 1977). En el caso de los instrumentos de participación ciudadana podríamos tratar de ordenar sus posibles funciones utilizando criterios similares.

En primer lugar, no hay duda que una característica de muchos de ellos es su capacidad de legitimar, como mínimo a las políticas concretas que se están adoptando y quizás en algunos casos también a aquellas 
instituciones que las generan. En segundo lugar, la representatividad ha sido uno de los temas de debate central cuando se habla de instrumentos participativos. En la tercera función hay mayores divergencias con los procesos electorales, ya que de la participación ciudadana en la toma de decisiones no debe salir ningún nuevo gobierno. Sin embargo, junto a legitimidad y representatividad, si deben conseguirse unos resultados. Estos rendimientos sustantivos del proceso pueden consistir a menudo en una decisión o un conjunto de recomendaciones, ideas o propuestas a las autoridades, pero en otras ocasiones el objetivo del proceso puede estar menos relacionado con la elaboración de políticas y más con mejorar algún aspecto concreto de la convivencia social y/o de la relación entre la sociedad civil y las administraciones públicas.

Los efectos legitimadores de los procesos participativos han sido estudiados de forma considerable en los últimos años(1), tanto desde los análisis más normativos, como desde el estudio de las experiencias más concretas. Aunque se trata básicamente de una legitimación procedimental, también sabemos que las características específicas de los procesos pueden contribuir a facilitar o dificultar esa función: un proceso basado en urnas, sufragio universal, con amplia visibilidad y elevada participación (Gómez y Font, 2015) resultará más fácilmente aceptable que uno basado en otras credenciales democráticas muy valoradas por los expertos pero no siempre tanto por la ciudadanía, como la selección de los participante a través de un sistema de sorteo (Font y Medina, 2001) o el énfasis en una deliberación muy rica.

Los problemas de falta de representatividad de los participantes constituyen un tema clásico de los estudios sobre participación, hasta el punto que los autores preocupados por el tema hayan llegado a proponer la participación obligatoria o el gobierno a base de encuestas (Verba, 1995), o el sistema de sorteo anteriormente mencionado, como únicas alternativas ante una objeción recurrente frente a casi cualquier mecanismo participativo: solo participa una minoría muy poco representativa de la sociedad (Fiorina, 1999). Sin duda, se trata de un tema que ha recibido un considerable tratamiento en el estudio de diversos mecanismos (por ejemplo, Ganuza y Francés, 2012) y ante el que no existe acuerdo entre los diversos autores ni en el diagnóstico ni en las soluciones. ¿Déficits de representatividad por desigualdad de recursos y de incentivos o estructuralmente inducidos por el propio diseño de los instrumentos participativos? ¿Queremos escuchar a tod@s o principalmete a los más afectad@s?

Aunque la participación tenga interés e importancia en sí misma, a la mayor parte de la ciudadanía le interesa especialmente hablar de ella en

(1) PAPADOPOULOS y WARIN (2007) coordinaron un número monográfico del European Journal of Political Research, en el que se analiza el papel de factores como la legitimidad y la eficacia. 
relación con la toma de decisiones colectivas. Por ello tiene sentido pensar que un tercer gran tema desde el que valorar los instrumentos participativos son sus resultados: ¿Sirven de verdad para tomar decisiones que se lleven a la práctica o son poco más que rituales donde escenificar un diálogo entre administradores y ciudadanía?

Esta propuesta de lectura de los criterios desde los que evaluar los procesos participativos es solo una de las posibles. Fung (2006) y Smith (2009) han hecho dos de las más influyentes(2). Fung (2006) propone un cubo democrático que también tiene tres dimensiones: quiénes son los participantes y qué nivel de poder tienen y cuáles son las formas de comunicar y decidir en el proceso. Por su parte, Smith (2009) menciona cinco grandes criterios: inclusividad, control popular, juicio informado, transparencia y eficiencia. Comparando las tres aproximaciones vemos algunas diferencias (la idea de legitimidad no es central para Fung y Smith opta por un listado de criterios más amplio y complejo) pero también importantes similitudes que pasan por tener muy en cuenta quién participa, cuáles son los resultados y la capacidad de influencia que poseen las personas participantes.

Podríamos hacer sin duda listados mucho más amplios y algo diferentes de cuáles serían los factores concretos a valorar. Pero, como hemos visto, los protagonistas y los productos del proceso participativo van a estar siempre en ellos. Dado que sabemos bastantes más cosas sobre los protagonistas, el resto de este texto estará dedicado a hablar sobre los potenciales resultados de los procesos participativos, empezando en el próximo apartado por su impacto en las políticas públicas.

\section{Procesos participativos y políticas públicas}

Dividimos esta sección en dos grandes partes. En la primera, revisaremos muy brevemente algunas de las evidencias empíricas con las que contábamos respecto a cuánto y cuándo influyen las propuestas generadas en los procesos participativos en las políticas finalmente adoptadas. En la segunda, sintetizaremos alguno de los principales resultados de un proyecto de investigación reciente en el que hemos abordado este tema para el caso español (3).

Una de las revisiones recientes del estado de la cuestión sobre el tema es bastante concluyente: los efectos parecen ser limitados y poco siste-

(2) Muchos otros autores han hecho también propuestas algo diferentes. En el contexto español podemos mencionar las de Jorba y Anduiza (2009), que propone indicadores concretos para cada una de las dimensiones de evaluación propuestas y la de Font y Blanco (2006).

(3) Para más información, visitar el blog del proyecto: https://cherrypickingproject.wordpress.com/ 
máticos (Mazeaud y otros, 2012). Efectivamente, miremos a donde miremos, la mayor parte de las aportaciones empíricas sobre el tema tienden a encontrar un reflejo cuando menos tenue de la voz de la ciudadanía en la adopción real de políticas. Ese es el diagnóstico si miramos a los municipios británicos (Lowndes y otros, 2001), pero también si ponemos el foco en la elaboración interactiva de políticas en Holanda (Tatenhove y otros, 2010), en los jurados ciudadanos españoles (Font y Medina, 2001), en la inmensa mayoría de encuestas deliberativas (Goodin y Drizek, 2006) o de conferencias de consenso danesas (Joss, 1998).

Para encontrar efectos algo más claros necesitamos poner el foco en otras experiencias, como por ejemplo algunos de los procesos de presupuestos participativos. En el caso más conocido, Porto Alegre, las evidencias favorables al impacto en las prioridades del presupuesto municipal son bastante amplias (Baiocchi, 2005). Aunque algunos de los estudios comparados más rigurosos apuntan a un escenario menos optimista, de efectos no sistemáticos (Boulding y Wampler, 2009).

Es precisamente la existencia de estas dudas sobre cuánto y cuando se produce este impacto de los procesos participativos en las políticas públicas lo que da pie a la aparición de la hipótesis de la escucha selectiva (Sintomer y otros, 2008), o del "cherry-picking", o la selección interesada de propuestas, que da lugar a la investigación que presentamos a continuación (4).

\section{El destino de las propuestas participativas}

Para evaluar la efectividad de los procesos participativos y su impacto en la elaboración de políticas públicas es necesario trazar el recorrido del conjunto de propuestas o recomendaciones surgidas tras su realización. Tanto el destino final como el trayecto de una propuesta participativa pueden ser múltiples. A continuación expondremos cuáles son algunos de los más comunes, aunque obviamente los recorridos reales no se agotan en ellos. Para ello, nos centraremos en los siguientes cuatro tipos de instrumentos de participación ciudadana: presupuestos participativos, planes estratégicos, mecanismos permanentes de participación ciudadana (principalmente consejos consultivos) y otros mecanismos puntuales (por ejemplo, encuestas ciudadanas sobre proyectos de inversión pública).

En el extremo ideal se puede dar la situación en la que una propuesta elaborada y aprobada en el contexto de un proceso participativo acabe

(4) Este proyecto ha contado con financiación del Ministerio de Economía y Competitividad (Convocatoria 2012) a quien agradecemos su apoyo. Extendemos la misma a los miembros del equipo de investigación, que han contribuido decisivamente a ir construyendo las ideas que aquí exponemos. 
implementándose completamente, es decir, tal y como quedó plasmada en el documento definitivo tras la finalización del proceso. Es probable que esta propuesta, antes de ser incluida en dicho documento definitivo, haya superado unos requisitos de viabilidad técnica y económica. Posteriormente, el conjunto de propuestas ha podido recibir incluso el apoyo y reconocimiento explícito por parte del gobierno responsable, quedando de ese modo recogido en sus actas y al alcance de la ciudadanía. Por último, y en el mejor de los mundos imaginables, ese gobierno puede llegar a informar durante alguna sesión plenaria del estado de ejecución de las propuestas. Sin embargo, en la mayoría de las ocasiones, el escenario más habitual dista enormemente del ejemplo que acabamos de exponer.

En el extremo contrario, encontraríamos que una propuesta o recomendación no llegue a ser implementada. En este caso, las autoridades locales o responsables del proceso participativo, pueden acompañar la no implementación de la misma con una explicación sobre el porqué de la paralización de la propuesta. Algunos autores subrayan que el hecho de que una propuesta no llegue al estado máximo de ejecución, no debe considerarse por sí mismo como una actitud poco democrática. Desde esta óptica, el acento se pone en la actitud transparente de las autoridades una vez finalizado el proceso. Si una propuesta después de haber sido aprobada y haber recibido el reconocimiento por parte de las autoridades, finalmente no es implementada - por ejemplo, por limitaciones presupuestarias sobrevenidas-, cabría esperar una explicación para que los participantes y el resto de la población pudiesen hacer sus valoraciones con el máximo de información posible.

Existe un aspecto importante a tener en cuenta y es el grado de adopción e implementación alcanzado por las propuestas. Por ejemplo, es posible que una propuesta haya sido formalmente aceptada e incluida en el programa de trabajo del departamento, pero finalmente no se haya implementado ni total ni parcialmente, debido por ejemplo a un cambio de gobierno.

La consideración sobre si una propuesta ha sido total o parcialmente implementada nos aproxima a otro aspecto crucial para entender el destino de las propuestas: saber si han sido modificadas y si se han explicado dichas modificaciones por parte de las autoridades. Esa modificación puede producirse en al menos dos sentidos: cuando la autoridad local cambia sustancialmente el contenido de la propuesta o cuando solo implementa una parte de ella.

En la siguiente sección expondremos cuáles son los potenciales factores explicativos que podrían explicar los diferentes resultados de estas propuestas. Es decir, ¿hay alguna lógica que nos permita entender por qué algunas son implementadas completamente, otras solo de forma parcial y un tercer grupo son completamente abandonadas? 


\section{Gráfico 1}

Posibles destinos de las propuestas surgidas de procesos participativos

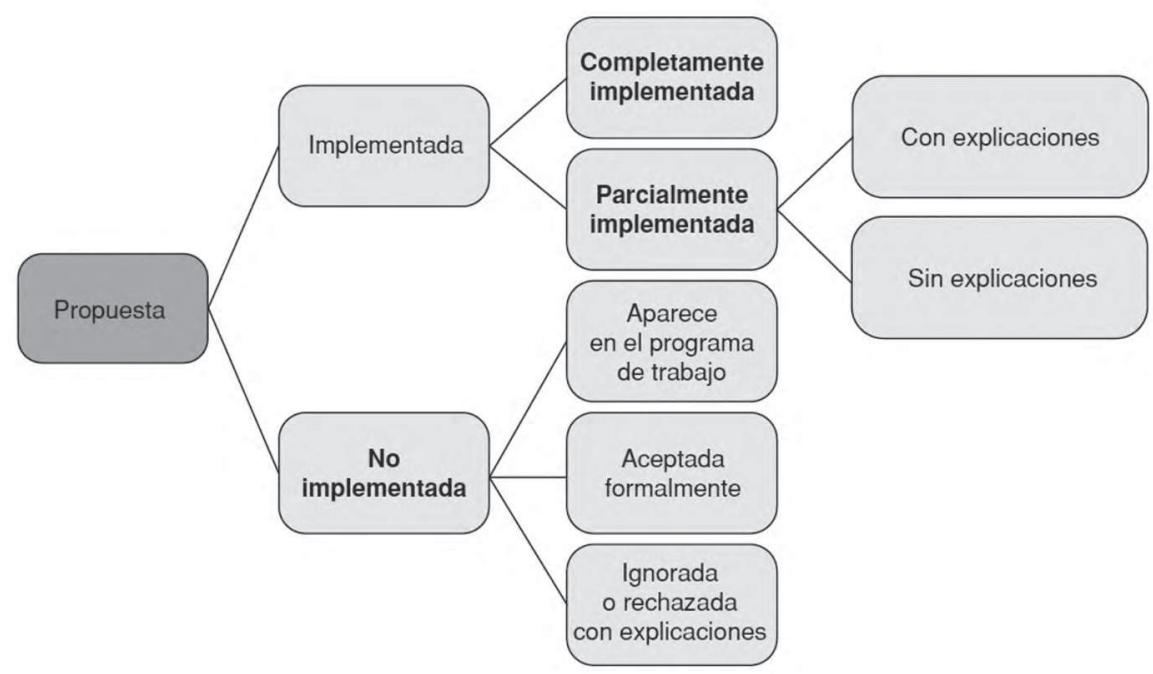

Fuente: elaboración propia.

\section{Posibles factores que influyen en la implementación}

La principal dificultad al analizar cuáles son las razones que explican la efectividad de un proceso participativo es cómo saber si sus efectos se deben a algún aspecto propio del diseño del instrumento participativo o a las propias dinámicas políticas y socioeconómicas en las que se encuentra inserto dicho instrumento. A continuación detallaremos los posibles factores que pueden ayudar a explicar el impacto de los procesos participativos en las políticas públicas. Para ello, clasificamos los factores en tres niveles de análisis atendiendo a las características del municipio, del proceso participativo y del contenido de las propuestas.

\section{En relación al municipio}

Se suele pensar que es más fácil desarrollar de manera exitosa mecanismos de participación ciudadana en pequeños municipios o en reducidas comunidades. Cabe esperar por tanto que en los municipios de tamaño reducido, la proximidad de los ciudadanos con las autoridades haga posible que exista una mayor presión para que las propuestas se Ileven finalmente a cabo. En segundo lugar, aquellos gobiernos locales 
con mayor acceso a recursos económicos, y humanos, son también aquellos que tienen más probabilidad de poder implementar las propuestas realizadas por la ciudadanía. Un tercer factor contextual está compuesto por aquellos aspectos relativos a la cultura organizacional del Ayuntamiento en relación a la participación, como pueden ser la tradición participativa y contar con un departamento y plan específico sobre participación ciudadana. Un cuarto factor sería la ideología o afiliación política del partido del gobierno. En este punto, existen evidencias que demuestran que existe más probabilidad que un proceso participativo, sobre todo, en el caso de los presupuestos participativos, sea impulsado por gobiernos de izquierdas. No obstante, estas evidencias sobre el inicio del proceso no pueden ser trasladadas de forma automática cuando lo que se aborda es el destino, o sea el grado de implementación de las propuestas. Por último, y en quinto lugar, hay que tener en cuenta el tiempo político, sobre todo el ciclo electoral. La proximidad con las próximas elecciones y la continuidad o cambio de gobierno pueden influir de manera determinante en el buen desarrollo y ejecución de los procesos participativos.

Tabla 1

Posibles factores que influyen en la ejecución de los procesos participativos

\begin{tabular}{|c|c|c|}
\hline \multirow{2}{*}{ 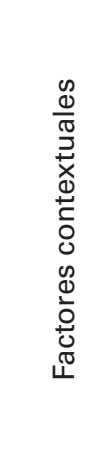 } & Relativos al municipio & $\begin{array}{l}\text { Tamaño del municipio } \\
\text { Recursos económicos } \\
\text { Cultura organizacional } \\
\text { Ideología del gobierno } \\
\text { Tiempo político }\end{array}$ \\
\hline & Relativos al proceso & $\begin{array}{l}\text { Institucionalización } \\
\text { Implicación de otras administraciones } \\
\text { Cantidad de propuestas } \\
\text { Calidad de proceso y la deliberación }\end{array}$ \\
\hline \multicolumn{2}{|c|}{ Factores relativos a la propuesta } & $\begin{array}{l}\text { Continuidad o cambios } \\
\text { Coste } \\
\text { Financiación } \\
\text { Competencias } \\
\text { Apoyo interno institucional }\end{array}$ \\
\hline
\end{tabular}




\section{En relación al diseño del proceso participativo}

Entre los aspectos relativos al diseño del proceso es preciso diferenciar, en primer lugar, entre el carácter temporal o permanente de los procesos. Cabe esperar que aquellos procesos más duraderos acaben adquiriendo un mayor grado de compromiso político y ello influya de manera positiva en la implementación de las propuestas (Morrell, 1999). Un segundo aspecto del proceso es la implicación de otras administraciones. Es previsible que aquellos procesos donde intervienen otras esferas de la administración, la presión por llevar a cabo las propuestas sea mayor (Fung, 2006). Un tercer factor a tener en cuenta es la cantidad de propuestas resultantes. Es razonable pensar que cuanto mayor sea la lista de propuestas, más probable es que las autoridades escojan entre ellas atendiendo a intereses propios. Otro elemento a destacar en este factor es la existencia y calidad de espacios de deliberación. Además de estos factores principales, existen otros a tener en cuenta: el carácter vinculante o no del proceso; quién es el impulsor del proceso (sociedad civil, administración o ambas); la visibilidad del proceso (medios de comunicación, redes sociales, etc.) y el tipo de participantes (asociaciones y/o ciudadanos a título Individual).

\section{En relación al contenido de las propuestas}

Una de las hipótesis principales que guía nuestro estudio recibe el nombre de cherrypicking. Esta se apoya en la idea de que no todas las propuestas surgidas de un mismo proceso van a recibir el mismo grado de reconocimiento por parte de las autoridades locales. En otras palabras, las autoridades locales acaban implementando unas propuestas, mientras que otras quedan paralizadas o pasan al olvido. Por tanto, siguiendo tal hipótesis, cabe esperar que el contenido de la propuesta sea determinante a la hora de predecir el destino de la misma. Unos de los elementos a tener en consideración es, precisamente, el carácter continuista de la propuesta en relación con lo que se venía desarrollando en un área concreta del ayuntamiento. En otras palabras, es previsible que aquellas propuestas que supongan un desafío o ruptura, alejándose de las líneas marcadas previamente por las autoridades, tengan menos probabilidad de ser aceptadas e implementadas. En segundo lugar, el coste de la propuesta es también un factor a no pasar por alto, del mismo modo que la existencia de diferentes fuentes de financiación. Un tercer elemento es quién tiene la competencia para llevar dicha propuesta a cabo. En ocasiones, una propuesta es formulada sin prever cuántos departamentos tienen competencia sobre esta propuesta, o incluso, si estas competencias 
están en manos de otras administraciones. Por último, es crucial saber cuál es el apoyo institucional que recibe una propuesta por parte del equipo de gobierno y el personal técnico. En procesos como los presupuestos participativos, la responsabilidad de desarrollarlos suele recaer en la concejalía que tiene atribuida la función de fomentar y canalizar la participación ciudadana. No obstante, en ocasiones, cuando el proceso ha finalizado se suele requerir la colaboración de otras áreas del Ayuntamiento que pueden mostrar resistencias al proceso o simple desconocimiento del mismo.

\section{Metodología}

Para profundizar en las causas que permiten explicar el diferente impacto de los procesos participativos en las políticas públicas a nivel local, establecimos como unidad de análisis en nuestra investigación aquellas propuestas surgidas de 39 procesos participativos desarrollados entre las elecciones locales de 2007 y las de 2011 en 25 municipios de Cataluña, Madrid y Andalucía. El punto de partida para seleccionar los procesos analizados fue la base de datos sobre mecanismos de participación local en el sur de Europa surgida de un proyecto anterior. El método de muestreo fue estratificado con el fin de asegurar la representación de diferentes tipos de mecanismos de participación y diferentes contextos socio-políticos. Las variables de estratificación fueron: la región, el número de procesos participativos desarrollados en el municipio y, por último, el tipo de proceso (Presupuestos participativos, Planificación Estratégica y otros mecanismos puntuales o permanentes). Los procesos participativos incluidos en el estudio se seleccionaron de manera aleatoria en cada uno de estos estratos.

Una vez seleccionados los procesos participativos se analizaron los documentos definitivos que contenían las propuestas y se procedió a su codificación. Se elaboró un cuestionario con todos los aspectos sobre los que se quería obtener información relativa al proceso y a cada una de las propuestas. La tarea de recogida de la información se llevó a cabo principalmente a través de entrevistas presenciales estructuradas realizadas la mayoría de ellas durante el año 2014. También se realizó una búsqueda de documentos oficiales y no oficiales que reflejasen el grado de implementación de una determinada propuesta. En total se entrevistaron a 181 informantes, principalmente técnicos del ayuntamiento, miembros de la oposición y participantes (5).

(5) Para más detalles de la metodología ver Font y otros (2016). 


\section{Grado de implementación de los procesos participativos}

Los resultados obtenidos al analizar el grado de implementación de las propuestas señalan dos conclusiones principales: 1) la mayoría de las propuestas (68 por 100 ) surgidas de procesos participativos acaban implementándose total o parcialmente; 2) existe una implementación selectiva de las propuestas, ya que en la mayoría de los procesos participativos las propuestas surgidas de un mismo proceso no corren la misma suerte (ver gráfico 3). Según los datos obtenidos, los procesos participativos parece que sí tienen impacto e influyen sobre las actuaciones concretas de los gobiernos y administraciones locales. En otras palabras, las autoridades locales no parece que permanezcan inmóviles frente a las propuestas sobre políticas públicas surgidas de este tipo de procesos.

No obstante, en línea con la hipótesis cherrypicking, en el 90 por 100 de los procesos analizados, existe una implementación selectiva de las propuestas. Sin duda, es preciso diferenciar por tipos de procesos cuál es el grado de implementación exacta alcanzada. En el gráfico 2 se pueden observar el grado alcanzado en cada uno de los 39 procesos analizados. Según los datos obtenidos, los presupuestos participativos y otros mecanismos permanentes, principalmente consejos consultivos o de barrio, destacan por una elevada implementación completa de las propuestas (58 y 62 por 100 respectivamente). Por el contrario, en el caso de los planes estratégicos y otros mecanismos temporales la implementación parcial representa aproximadamente una tercera parte (37 y 27 por 100 respectivamente). 


\section{Gráfico 2}

Nivel de implementación por proceso participativo.

Agenda 21

Agenda 21

Agenda 21

Agenda 21

Plan Estratégico

Plan Estratégico

Proyecto Iniciativa Urbana

Proyecto Educativo de Ciudad

Plan Reavivación del Barrio Antiguo

Plan para Disenar un Parque

Plan Municipal de Participación Ciudadana

Plan Municipal de Igualdad Plan de Movilidad Urbana Sostenible

Plan sobre Ahorro Energético

Presupuestos Participativos

Presupuestos Participativos

Presupuestos Participativos

Presupuestos Participativos

Presupuestos Participativos

Presupuestos Participativos Infantil Presupuestos Participativos Infantil Sugerencias al Presupuesto Municipal Consejo de Bienestar Social Consejo de la Infancia Consejo de Mujeres Consejo de Mujeres Consejo Municipal de la Salud Consejos de Barrio Consejo de Seguridad Consejo Municipal

Proceso para la Mejora de una Plaza Proceso para la remodelación de un barrio Proceso para la Creación de unas Mesas Territoriales Consulta Ciudadana Plan de Inversiones Proyecto de Ocio Alternativo Juvenil Foro Local sobre Inmigración Foro para la Creación de un Consejo Sectorial Proceso para la Construcción de un Paso a Nivel Propuestas sobre Presupuestos Participativos

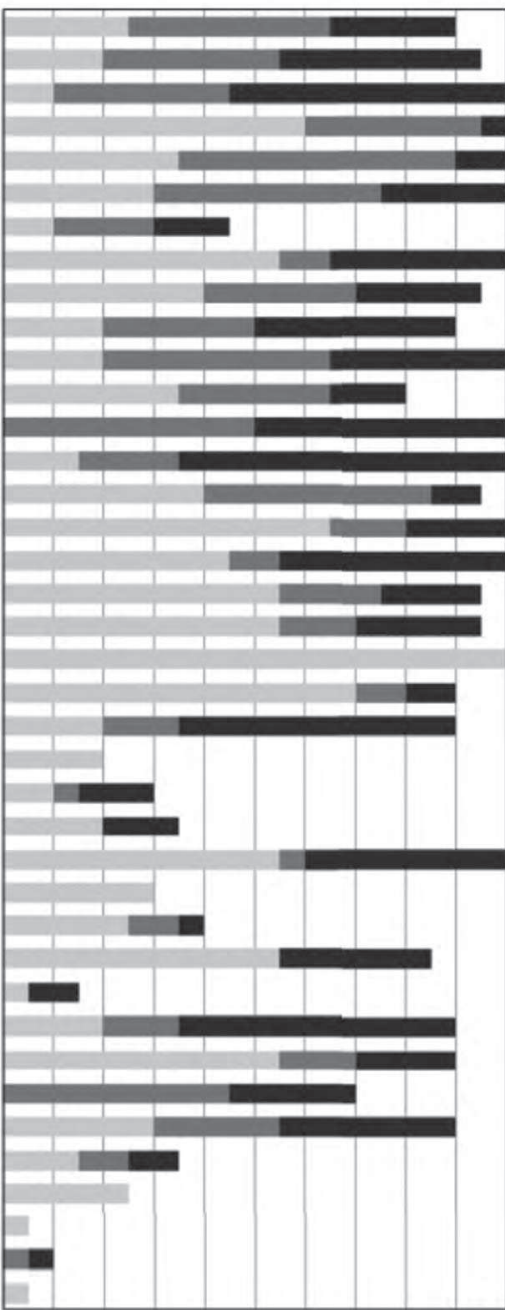

$\square$ Completamente implementada $\square$ Parcialmente implementada $\quad$ Rechazada

Fuente: Base de datos del proyecto Cherrypicking. 


\section{El proceso de rendición de cuentas tras los procesos participativos}

Otras de las cuestiones a tener en cuenta están relacionadas con el grado de compromiso que las autoridades públicas adquieren con los procesos participativos (authority) y con el proceso de rendición de cuentas (accountability) cuando las propuestas no son finalmente implementadas. Es decir, ¿una vez finalizado el proceso participativo, las autoridades se comprometen con los resultados del mismo? ¿Existe obligación de cumplir con lo surgido de ese proceso?Y más allá del proceso en su conjunto ¿hay determinadas propuestas que habiendo sido o bien rechazadas o bien modificadas, reciben algún tipo de explicación por parte de las autoridades o administración locales? ¿Cómo se produce esa explicación? $\mathrm{O}$ en caso contrario ¿qué razones - basadas tanto en hechos objetivos como en actitudes y valores - hay detrás del hecho de no dar explicaciones?

En la actualidad existen mecanismos de participación que poseen un alto grado de authority, (referéndum, iniciativa legislativa o plebiscito). En estos casos, a veces se requiere un específico número o porcentaje de ciudadanos para que sea de obligado cumplimiento (Smith, 2009). No obstante, los instrumentos de participación ciudadana que son objeto de nuestro estudio no suelen alcanzar ese grado tan elevado de authority. De los mecanismos participativos analizados, los que generan una mayor authority son los presupuestos participativos y seguidamente, los planes estratégicos. En el caso de los presupuestos participativos la ciudadanía se implica a través de una serie de asambleas y votaciones eligiendo el destino de un porcentaje del presupuesto municipal, correspondiente habitualmente al capítulo de inversiones. El compromiso adquirido suele ser principalmente unidireccional: las autoridades se comprometen a realizar las propuestas surgidas de los presupuestos participativos y que sean viables, por ejemplo, desde un punto de vista técnico. En el caso de los planes estratégicos, el compromiso de las autoridades públicas se establece en varias direcciones y niveles. Por ejemplo, con organismos nacionales o supranacionales que subvencionan a través de un programa concreto (medioambiental, movilidad, igualdad, etc.) unas líneas de actuación determinadas.

Por otra parte, los consejos consultivos son sin duda el mecanismo más extendido en España. En la mayoría de los municipios medianos y grandes se pueden encontrar distintos tipos de consejos consultivos sectoriales (consejo de la mujer, de la infancia, de comerciantes, de la juventud). En cambio, el nivel de compromiso adquirido por este tipo de instrumentos es, en muchas ocasiones, puramente simbólico. Algo similar ocurre con las experiencias participativas puntuales, en las que después de un gran esfuerzo de movilización por parte del personal técnico del 
ayuntamiento para que participe el mayor número posible de ciudadanos, apenas existe posteriormente un compromiso explícito o ronda de explicaciones sobre los resultados finales del proceso.

La rendición de cuentas (accountability) ha recibido especial atención en el mundo anglosajón. No obstante, en el contexto español estas prácticas basadas en dar explicaciones a los ciudadanos, en el marco de los procesos participativos, han recibido escasa atención. Ello se debe, principalmente, a que hasta ahora se ha puesto mayor énfasis en la descripción del proceso (perfil de los participantes, tipos de metodologías empleadas durante el proceso, aprendizajes adquiridos por los ciudadanos, etc.), que en el análisis de los resultados y efectos de dichos mecanismos de participación.

Si analizamos el proceso de accountability durante la fase de aceptación e implementación de las propuestas, observamos que entre aquellas propuestas que han sido implementadas de manera parcial (26,3 por 100), bien porque solo se ha llevado a cabo una parte o porque la propuesta ha sufrido una modificación importante, el 64,1 por 100 de ellas no ha venido acompañada de una explicación, ya sea por parte de los técnicos municipales o de las autoridades políticas. En el caso de aquellas propuestas finalmente no implementadas (31,2 por 100), solo el 42 por 100 de ellas han sido rechazadas activamente, es decir con algún tipo de explicación, mientras que el resto podemos decir que han sido ignoradas.

\section{Gráfico 3}

Nivel de accountability detrás de los procesos participativos.

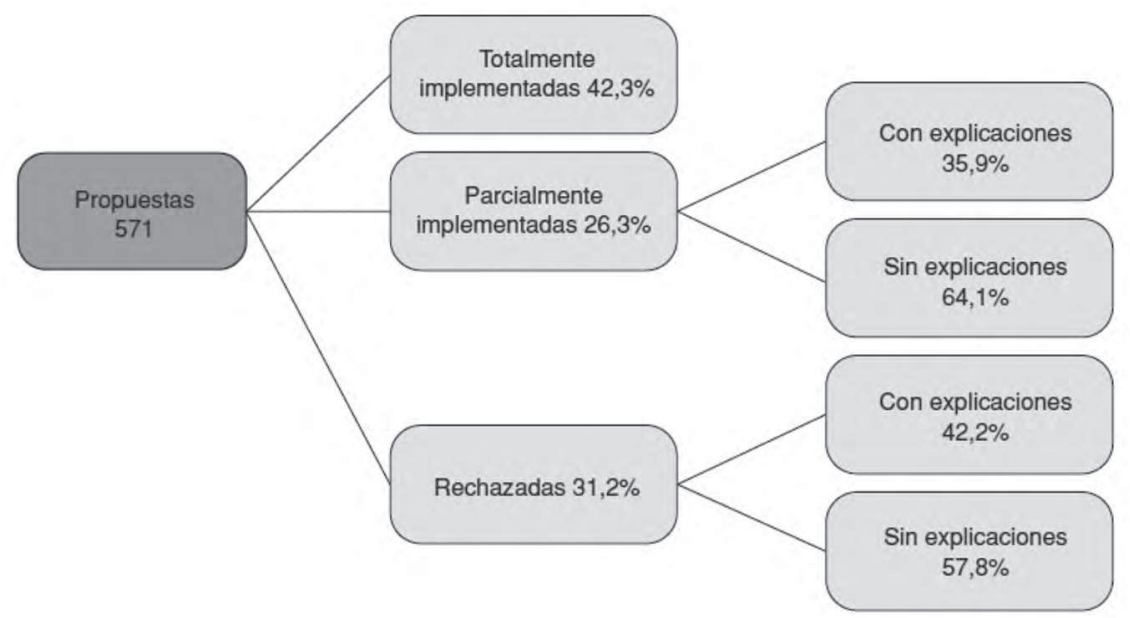

Fuente: Base de datos del proyecto Cherrypicking. 
En definitiva, las propuestas surgidas de procesos participativos locales en España se implementan algo más de lo que las limitadas investigaciones previas parecían apuntar, aunque ello pueda deberse a que muchas de estas propuestas son muy modestas en cuanto a lo que proponen y al nivel de ruptura política y administrativa que suponen. En todo caso, las políticas públicas no lo son todo $y$, por ello, dedicamos la próxima sección a analizar las consecuencias culturales que tienen estos mismos procesos participativos.

\section{Cambios en el contexto político local}

Los procesos participativos también tienen efectos sobre el contexto o comunidad política (polity). Estas instituciones introducen cambios en los actores políticos y en sus modos de interacción. Los procesos participativos suelen ponerse en práctica con objetivos relacionados con la democratización del contexto político local (Fung, 2009; Baiocchi et al., 2008). Ésta a menudo se vincula al objetivo de incrementar las capacidades de decisión, gestión, influencia política y recursos para la acción colectiva de los sujetos participantes y especialmente de los miembros de entidades asociativas (Fagotto y Fung, 2006; 2009), como principales actores involucrados en estos espacios (Alarcón y Font, 2014).

Las consecuencias democratizadoras han sido abordadas desde distintas perspectivas. En primer lugar, se han estudiado los cambios culturales y aprendizajes cívicos que adquieren los sujetos participantes. Por ejemplo, a través de un estudio en experiencias de presupuesto participativo, Talpin (2012) identifica cuatro vías de transformación: a) los participantes que acaban cayendo en la frustración política; b) aquellos que se convierten en "ciudadanos expertos" con amplios conocimientos sobre el funcionamiento de la administración; c) otros que se introducen en el mundo del activismo social a raíz de su experiencia; y d) otros que son cooptados o ascienden dentro de los partidos políticos locales. Otros estudios han desarrollado las competencias cognitivas, cívicas o relacionales que los participantes adquieren tras la participación en estos procesos (Talpin, 2012b; Pincock, 2012; Talpin, Funes y Rull, 2014; Funes, 2016).

Además de los cambios en los sujetos, la literatura empírica ha puesto el foco en los cambios en los grupos de la sociedad civil y en su relación con las autoridades públicas. ¿Son las instituciones participativas un «revulsivo" para el capital social local? En un trabajo reciente (García Espín y Jiménez Sánchez, 2015), planteamos tres procesos de cambio en los contextos políticos locales. En primer lugar, planteamos la hipótesis del arrecife de coral, según la cual las instituciones participativas facilitarían la aparición de nuevos actores asociativos, la sociabilidad y la coordinación 
entre grupos. En segundo lugar, desarrollamos la hipótesis del empoderamiento, según la cual las instituciones participativas generan nuevos recursos para los colectivos sociales (recursos materiales, informativos, relacionales, poder e influencia, etc.). Finalmente, desarrollamos la hipótesis del cambio en las relaciones de poder que comprende las trasformaciones en la relación entre las autoridades públicas y las entidades asociativas.

En este apartado, nos concentraremos en esos cambios en las relaciones entre autoridades públicas y entidades asociativas que produce la puesta en marcha de instrumentos participativos. En algunos casos esta dimensión ha sido objeto de análisis empírico. Por ejemplo, Baiocchi et al. (2008; 2011) analizan en el marco de presupuestos participativos en Brasil cómo cambian las relaciones entre actores políticos locales. En esos contextos, tendían a producirse nuevos escenarios caracterizados por un clientelismo autónomo, donde los actores asociativos mantienen una independencia formal frente al gobierno, al tiempo que cultivan una relación clientelar subordinada a los intereses electorales de esos gobiernos. En otros casos donde existían previamente movimientos sociales y entidades asociativas poderosas, los presupuestos participativos abrieron escenarios de autonomía empoderada. En ellos, las entidades asociativas ganaron influencia política y mantuvieron su autonomía y nivel de movilización previo. La introducción de instituciones participativas ambiciosas (como los presupuestos participativos) puede dar lugar a cambios significativos en las relaciones entre actores. Ello depende del diseño participativo y de la configuración previa de las entidades asociativas.

A continuación, exponemos los resultados de un estudio comparativo de seis casos que aborda este tipo de cambios. Los detalles precisos de ese estudio pueden verse en Bherer et al., 2016 y en la web del proyecto Cherrypicking (6). Para reconstruir los efectos de los procesos participativos en el contexto político y en las relaciones entre actores, seleccionamos seis casos: tres presupuestos participativos y tres consejos consultivos (dos en Andalucía, dos en Madrid y dos en Cataluña). El criterio central de la muestra era seleccionar experiencias intermedias: dejamos de lado aquellas definidas como "buenas prácticas" por la literatura y las experiencias con más recursos (ciudades más grandes), al tiempo que seleccionábamos experiencias con una mínima calidad (que contaran con espacios deliberativos, con influencia sobre las políticas y que hubieran durado más de un año). El estudio de casos «intermedios» nos permitía situarnos en experiencias de calidad intermedia, distanciándonos de la

(6) En esta sección se pueden ver los detalles de la metodología empleada: https://cherrypickingproject.wordpress.com/2016/02/12/the-back-office-methods-cases-data/ 
tendencia de la literatura a concentrarse en experiencias exitosas, donde se dan cambios más espectaculares. No situábamos así en experiencias más modestas, reflejando un sector importante de los instrumentos participativos que se ponen en marcha en el contexto español. La Tabla 2 muestra las características básicas de los casos estudiados. En cada uno de ellos, entrevistamos a 9 informantes clave del ámbito de la representación pública, trabajadores de la administración y miembros de entidades asociativas. A continuación, describimos cuatro procesos de cambio en la comunidad política y en las relaciones entre actores que observamos en los casos estudiados.

Tabla 2

Principales características de los casos estudiados*

\begin{tabular}{|c|c|c|c|c|}
\hline Casos & $\begin{array}{l}\text { Contexto y } \\
\text { duración }\end{array}$ & Quién participa & $\begin{array}{l}\text { Principales } \\
\text { instituciones }\end{array}$ & Productos \\
\hline $\begin{array}{l}\text { PP- Andalucía } \\
\text { Presupuesto } \\
\text { participativo }\end{array}$ & $\begin{array}{l}\text { Ciudad } 100.000 \\
\text { habitantes aprox. } \\
\text { CCAA Andalucía } \\
\text { 2008-2011 }\end{array}$ & $\begin{array}{l}\text { Abierto a toda la } \\
\text { ciudadanía } \\
100-200 \\
\text { participantes }\end{array}$ & $\begin{array}{l}\text { Asambleas de } \\
\text { auto-regulación } \\
\text { Grupos motores } \\
\text { Asambleas de } \\
\text { zona } \\
\text { Consejo de } \\
\text { Ciudad }\end{array}$ & $\begin{array}{l}\text { Propuestas para } \\
\text { el presupuesto } \\
\text { municipal } \\
\text { Estimado en } \\
€ 230,000 \text { en } 2009 \\
\text { Carácter vinculante } \\
\text { Baja implementación } \\
\text { final }\end{array}$ \\
\hline $\begin{array}{l}\text { PP- Madrid } \\
\text { Presupuesto } \\
\text { participativo }\end{array}$ & $\begin{array}{l}\text { Ciudad de } \\
150.000 \\
\text { habitantes aprox. } \\
\text { CCAA Madrid } \\
\text { 2004-2011 }\end{array}$ & $\begin{array}{l}\text { Abierto a toda la } \\
\text { ciudadanía } \\
\text { De } 1004(2004) \\
\text { a } 3939 \text { (2010) } \\
\text { participantes }\end{array}$ & $\begin{array}{l}\text { Mesas } \\
\text { territoriales } \\
\text { Consejo de } \\
\text { Coordinación } \\
\text { Asambleas de } \\
\text { zona }\end{array}$ & $\begin{array}{l}\text { Propuestas para } \\
\text { el presupuestos } \\
\text { municipal } \\
1.2 \text { mill. } €(2004), 3 \\
\text { mill. } €(2010)\end{array}$ \\
\hline $\begin{array}{l}\text { PP- Cataluña } \\
\text { Presupuesto } \\
\text { participativo }\end{array}$ & $\begin{array}{l}\text { Ciudad de } \\
200.000 \\
\text { habitantes aprox. } \\
2008-2011\end{array}$ & $\begin{array}{l}\text { Abierto a toda } \\
\text { la ciudadanía } \\
\text { (votación final de } \\
\text { propuestas) } \\
\text { Entidades } \\
\text { asociativas y } \\
\text { ciudadanos elegido } \\
\text { por sorteo (mesas } \\
\text { territoriales) }\end{array}$ & $\begin{array}{l}\text { Mesas } \\
\text { territoriales } \\
\text { Consejo de } \\
\text { Coordinación } \\
\text { Proceso de } \\
\text { votación final }\end{array}$ & $\begin{array}{l}\text { Propuestas para } \\
\text { el presupuesto } \\
\text { municipal de } \\
\text { inversiones } \\
4 \text { millones de euros } \\
\text { (2009) }\end{array}$ \\
\hline
\end{tabular}

* Elaboración propia 
Joan Font Fàbregas, José Luis Fernández-Martínez y Patricia García Espín Instrumentos para la participación ciudadana y requisitos para su efectividad

\begin{tabular}{|c|c|c|c|c|}
\hline Casos & $\begin{array}{l}\text { Contexto y } \\
\text { duración }\end{array}$ & Quién participa & $\begin{array}{l}\text { Principales } \\
\text { instituciones }\end{array}$ & Productos \\
\hline $\begin{array}{l}\text { CC-Andalucía } \\
\text { Consejo } \\
\text { Consultivo de } \\
\text { Voluntariado }\end{array}$ & $\begin{array}{l}\text { Ciudad de } \\
200.000 \\
\text { habitantes aprox. } \\
\text { CC.AA Andalucía } \\
\text { 2007- continúa }\end{array}$ & $\begin{array}{l}\text { Stakeholders, } \\
\text { miembros } \\
\text { de entidades } \\
\text { asociativas de } \\
\text { distintos sectores } \\
\text { (renovación } \\
\text { cada } 4 \text { años) y } \\
\text { representantes } \\
\text { públicos (partidos } \\
\text { políticos) } \\
\text { 19-35 participantes }\end{array}$ & $\begin{array}{l}\text { Consejo y } \\
\text { comisiones } \\
\text { Frecuentes } \\
\text { reuniones }\end{array}$ & $\begin{array}{l}\text { Consejo consultivo } \\
\text { con autoridad } \\
\text { extendida } \\
\text { Iniciativa y } \\
\text { codecisión en las } \\
\text { actividades } \\
\text { Restringido al ámbito } \\
\text { de voluntariado }\end{array}$ \\
\hline $\begin{array}{l}\text { CC- Madrid } \\
\text { Consejo de } \\
\text { Entidades } \\
\text { Asociativas }\end{array}$ & $\begin{array}{l}\text { Ciudad de } 45.000 \\
\text { habitantes aprox. } \\
\text { CC.AA Madrid } \\
\text { 2002- continúa }\end{array}$ & $\begin{array}{l}\text { Representantes } \\
\text { públicos (partidos } \\
\text { políticos) y } \\
\text { entidades } \\
\text { asociativas } \\
\text { (vecinales y otras) } \\
\text { 13-19 participantes }\end{array}$ & $\begin{array}{l}\text { Consejo y } \\
\text { comisiones } \\
2-5 \text { reuniones por } \\
\text { año }\end{array}$ & $\begin{array}{l}\text { Consejo consultivo } \\
\text { Función consultiva y } \\
\text { recomendaciones al } \\
\text { pleno municipal }\end{array}$ \\
\hline $\begin{array}{l}\text { CC-Cataluña } \\
\text { Consejo de } \\
\text { Entidades } \\
\text { Asociativas }\end{array}$ & $\begin{array}{l}\text { Ciudad de } 45.000 \\
\text { habitantes aprox. } \\
\text { CC.AA Cataluña } \\
\text { 2006-2011 }\end{array}$ & $\begin{array}{l}\text { Representantes } \\
\text { públicos (partidos } \\
\text { políticos), } \\
\text { representantes } \\
\text { de una variedad } \\
\text { de entidades } \\
\text { asociativas de } \\
\text { distintos tipos } \\
\text { y ciudadanos } \\
\text { elegidos por sorteo } \\
40-50 \text { participantes }\end{array}$ & $\begin{array}{l}\text { Consejo y talleres } \\
\text { monográficos } \\
1-2 \text { reuniones por } \\
\text { año }\end{array}$ & $\begin{array}{l}\text { Consejo consultivo } \\
\text { Función consultiva y } \\
\text { recomendaciones al } \\
\text { pleno municipal }\end{array}$ \\
\hline
\end{tabular}

Fuente: elaboración propia.

\section{Incipiente pluralidad y sociabilidad en la red de políticas}

Tras poner en marcha procesos participativos, el primer cambio suele afectar a la composición de la red de políticas públicas. Así, pueden facilitar la creación de nuevos grupos asociativos o la incorporación de actores asociativos que antes permanecían excluidos de la red de políticas públicas En este sentido, la institución participativa puede representar una oportunidad nueva y significativa para algunos actores que no dudan en aprovecharla. Wampler (2007), en su estudio de presupuestos participativos en Brasil, plantea que estos procesos pueden propiciar la incorporación de nuevos actores incrementando la pluralidad en la red de políticas, al incluirse nuevos intereses y reivindicaciones. Esos incrementos 
en la pluralidad pueden ser más o menos duraderos: pueden perdurar después de la desaparición del proceso participativo, extenderse a otras áreas o desvanecerse una vez se suprime el proceso.

En los estudios de caso realizados en consejos consultivos y presupuestos participativos, observamos que los incrementos en la pluralidad de las redes de políticas se daban fundamentalmente en los segundos. Éstos tuvieron un efecto atracción y representaron una oportunidad de influencia para algunos colectivos sociales que anteriormente no se relacionaban con las autoridades públicas. Por ejemplo, en los tres presupuestos participativos observamos que nuevos colectivos se crearon al calor del proceso. En el caso de Andalucía (PP- Andalucía), un colectivo de inmigrantes sin papeles se organizó para presentar una propuesta de banco del tiempo, para intercambiar servicios con los vecinos. Los dinamizadores del proceso participativo y miembros de una ONG impulsaron esa iniciativa conjuntamente con el colectivo de inmigrantes. En otros dos casos de presupuestos participativos, se crearon nuevos grupos de jóvenes skaters con el objetivo de realizar sus propuestas. Para estos colectivos, los presupuestos participativos (que garantizan el carácter vinculante de las propuestas aprobadas) significaron una oportunidad política significativa que motivaba su incorporación en los procesos.

En los consejos sectoriales no observamos tal incorporación de nuevos actores a los procesos. En ese sentido, no son tan atrayentes e impulsores de la pluralidad en la red de políticas. Sin embargo, observamos una mayor sociabilidad entre autoridades públicas y actores asociativos. Se incrementaba el contacto, la relación y, posteriormente, ello facilitaba la relación en otras áreas de política públicas o en situaciones futuras. En este sentido, por ejemplo, en un consejo de entidades asociativas (CC-Madrid), miembros de entidades manifestaban cómo el hecho de participar en ese espacio suponía un mayor contacto con las autoridades de distintas áreas («intercambiamos teléfonos»), y ello favorecía el contacto posterior de cara a formular otras demandas o a colaborar en futuras situaciones de necesidad o conflicto. Así, el elemento más básico de la red de políticas (los actores y su contacto) quedaba ligeramente alterado por la introducción de estos instrumentos participativos, en el sentido de promocionar una ligera mayor sociabilidad.

\section{Cambios en los flujos de información y en la transparencia}

Cuando se ponen en marcha instituciones participativas, el segundo cambio que observamos es la alteración en los flujos de información. El 
modelo de comunicación habitual estaría caracterizado por los flujos de tipo vertical (la información es ofrecida por la autoridad pública o la administración, de acuerdo con su propio criterio), bilateral y selectiva (es compartida con una selección de grupos asociativos). En primer lugar, la puesta en práctica de procesos participativos podría dar lugar a un aumento de la información vertical. En segundo lugar, también podría alterar la dirección de los flujos de información, en un sentido multilateral (en espacios abiertos compartidos por una diversidad de entidades). Ello daría lugar a una mayor transparencia en la gestión de la información y, consecuentemente, incrementaría las capacidades para controlar la actividad de las autoridades públicas (Wampler, 2004).

En los casos estudiados, el aumento de la información es uno de los primeros efectos que se dan como consecuencia de la puesta en marcha de instituciones participativas. Por ejemplo, en dos casos de consejos consultivos (CC- Madrid y Cataluña), los miembros de entidades asociativas subrayaban ese mayor flujo de información sobre la actividad del Ayuntamiento. Esto era valorado positivamente por los representantes de esas entidades. Sin embargo, el aumento de la información no alteraba la dirección vertical de la misma. Por ejemplo, en el consejo de entidades asociativas de Madrid: a) el Ayuntamiento informaba sobre algunos de sus proyectos a las entidades asociativas presentes en el consejo; $b$ ) las entidades hacían peticiones de información de acuerdo con sus intereses particulares $y$, sobre todo, respecto a sus propios proyectos y demandas; y c) las autoridades respondían ofreciendo información extra al respecto. Las entidades asociativas perciben esta institución como un espacio donde adquirir nuevas informaciones sobre la actividad municipal, pero perciben escasa influencia política sobre esas decisiones y proyectos públicos.

En otro caso de consejo consultivo se observa además una forma más multilateral y horizontal de gestión de la información. Las autoridades públicas y los actores asociativos comparten información de manera multilateral y abierta sobre sus respectivas actividades. Por ejemplo, en el consejo sectorial de voluntariado (CC- Andalucía), los representantes de las entidades asociativas manifestaban que habían empezado a compartir información sobre sus agendas fuera del consejo sectorial. Esto redundaba en un incremento en la coordinación de actividades públicas y privadas en el sector. La causa fundamental de este cambio en los modos de distribuir la información se derivaba de la amplia presencia de entidades en el consejo (la mitad de los participantes) y de la autoridad que éstas habían ganado en las deliberaciones y decisiones en ese espacio. Ello les daba confianza para profundizar en la coordinación y la transferencia de información más allá de las actividades precisas del consejo. 
En los casos de presupuestos participativos, observamos cómo la gestión de la información cambiaba hacia fórmulas basadas en la apertura y la transparencia. Por ejemplo, en el caso seleccionado en Madrid (PP- Madrid) se puso en marcha una web en la que se podía hacer seguimiento de toda la política pública, sus fases, los espacios, las propuestas y los ritmos de implementación de las mismas. La web permitía el acceso a una cantidad significativa de información sobre esa política. Esa apertura y acceso abierto facilitaba, a su vez, un mayor control y seguimiento por parte de los miembros de entidades asociativas. Una vez se suprime ese presupuesto participativo en 2011, esas prácticas cotidianas desaparecen; pero los representantes de entidades asociativas mantienen una memoria satisfactoria de una gestión de la información más transparente.

\section{Institucionalización incipiente de nuevos canales basados en la autoridad}

En los casos estudiados, también observamos cambios en la ubicación de la autoridad y en la capacidad decisoria de los participantes. Tras la introducción de estas instituciones, las entidades asociativas involucradas y sus miembros podrían incrementar su capacidad decisoria en la red de políticas. Esa creciente autoridad puede producirse tanto en términos formales (por ejemplo, las decisiones son vinculantes y se implementan efectivamente), como en términos culturales, en contextos participativos donde las propuestas no son vinculantes pero los actores adquieren poder de decisión. Sólo en dos de los seis casos estudiados observamos cambios relevantes en términos de autoridad y capacidad decisoria, de acuerdo con los sujetos entrevistados.

En uno de los casos estudiados (CC-Andalucía) se había dado un incremento progresivo de la autoridad de las entidades asociativas. Ello se debía, en primer lugar, al diseño de ese consejo de voluntariado que establecía la paridad entre participantes de entidades asociativas y autoridades públicas. También se había realizado un pre-proceso participativo, por lo que las reglas del consejo habían sido establecidas contando con las aportaciones de las entidades asociativas. Finalmente, la escasez de medios en el contexto de crisis, hará que las entidades adquieran más protagonismo e iniciativa sobre las políticas adoptadas y en la provisión de recursos para implementarlas. El papel y capacidades decisorias, de gestión y control de las entidades irán ganando peso como consecuencia de esas dinámicas formales e informales. De hecho, toda la política de voluntariado que realiza el Ayuntamiento es supervisada y, frecuentemente, iniciada por el propio consejo consultivo siguiendo una lógica de coproducción de políticas. 
En otro de los casos estudiados de presupuesto participativo (PPMadrid) también observamos un cambio en la distribución y lugares de la autoridad. En ese presupuesto participativo, que duró de 2004 a 2011, las asambleas territoriales adquirieron una autoridad importante en la definición de la agenda política territorial y barrial. Así, en 2011, en las elecciones locales, los partidos políticos usaron las propuestas procedentes de las asambleas de presupuesto participativo para construir sus programas electorales. Como explicaba un técnico de participación, éstas asambleas "ofrecían un mapa de las necesidades de la ciudad". La atención y reconocimiento hacia las propuestas daba cuenta de la autoridad cultural que habían adquirido las asambleas territoriales en la definición de la agenda local. Los representantes de entidades asociativas mostraban esa memoria de "autoridad adquirida». Este proceso no sólo se basaba en el reconocimiento de la autoridad formal (carácter vinculante de las decisiones) sino que, tras varias ediciones, los participantes y particularmente los miembros de entidades habían alcanzado también un grado importante de autoridad cultural a través de las asambleas de zona. El carácter vinculante del presupuesto, así como su duración extendida contribuyeron a significar ese proceso institucional como una oportunidad para una influencia política efectiva.

\section{Prácticas clientelares dentro y fuera del espacio participativo}

Los tres procesos de cambio en el contexto político local (en la polity) encontraron, no obstante, obstáculos en su desarrollo. Las relaciones que tienden a introducir los procesos participativos estudiados no acaban de desarrollarse en la mayoría de los casos estudiados (4 de 6). Uno de los obstáculos más relevantes es la presencia de redes y prácticas clientelares. Definimos las prácticas clientelares como una suerte de pork-barrel politics (Ferejohn, 1974), un conjunto de relaciones de intercambio que se dan entre las autoridades públicas y una selección de entidades asociativas. Esas relaciones están caracterizadas por la selectividad (vs. pluralidad), la bilateralidad (vs. multilateralidad), y la recreación de coaliciones electorales en las que el partido gobernante suele dominar el marco. Este tipo de relaciones e intercambios perviven dentro de los procesos participativos y alrededor de ellos, generando resistencias y conflictos en el despliegue de los nuevos cauces más plurales, transparentes y empoderados.

Esas prácticas resultan especialmente conflictivas con los presupuestos participativos, que son los que incluyen procedimientos más ambiciosos en los términos antes señalados. Por ejemplo, en un presupuesto 
participativo (PP-Andalucía), se ponía en marcha un esquema de institucional basado en asambleas de zona para realizar y priorizar propuestas en diversas materias (obras menores, mantenimiento, cultura, juventud, etc.). Este proceso participativo había sido impulsado por Izquierda Unida (socio minoritario en cogobierno), mientras que el PSOE mantenía un compromiso menor con ese instrumento. En la segunda edición del presupuesto participativo, un conjunto de representantes vecinales - que formaban parte de una de las federaciones de AA.VV. de la ciudad-publican un manifiesto para mostrar su oposición. En ese manifiesto reivindican que las entidades vecinales debían tener un reconocimiento expreso de su papel representativo (una especie de voto cualificado u otro tipo de posición representativa) dentro de las asambleas y órganos del esquema institucional. Varios representantes de estas entidades esgrimen que ellos ya mantenían relaciones bilaterales estrechas con el Ayuntamiento, por lo que el presupuesto participativo no suponía una alternativa atractiva para influir en las políticas. Esas relaciones preferentes, bilaterales y opacas se van a seguir manteniendo al margen del proceso participativo. Ello es interpretado por otros representantes de entidades asociativas, por técnicos y observadores como una estructura paralela de relaciones opacas y clientelares que obstaculizan la consolidación de los nuevos procesos participativos abiertos, multilaterales y vinculantes.

En otro caso de presupuesto participativo (PP-Cataluña), los intercambios de tipo clientelar se practicaron dentro del mismo proceso participativo, generando dinámicas de conflicto. En el PP-Cataluña se aprobaron en votación final 10 propuestas de obras públicas. De acuerdo con los representantes de entidades asociativas y otros perfiles técnicos y políticos, el gobierno municipal priorizó la implementación de aquellas propuestas procedentes de entidades vecinales con las que mantenía una vinculación más estrecha basada en afinidades electorales. En ese proceso de priorización (o "cherry-picking", como explicábamos más arriba), el gobierno desechó la propuesta de un colectivo de jóvenes skaters para la construcción de un skate-park en un espacio público. Aunque el gobierno justificó ese rechazo por las restricciones presupuestarias sobrevenidas, ello fue interpretado por los representantes de un sector de entidades asociativas como un sesgo en la implementación de propuestas. El incumplimiento de propuestas y el sesgo en la implementación contribuyeron de manera decisiva a los sentimientos de frustración tras el proceso participativos y a su no consolidación. 
Tabla 3

Cambios detectados en los casos estudiados

\begin{tabular}{|c|c|c|c|c|c|c|}
\hline & PP- Andalucía & PP- Madrid & PP- Cataluña & CC-Andalucía & CC- Madrid & CC- Cataluña \\
\hline $\begin{array}{l}\text { Pluralidad y } \\
\text { apertura }\end{array}$ & $\begin{array}{l}\text { Nuevos } \\
\text { actores en } \\
\text { la red de } \\
\text { políticas }\end{array}$ & Nuevos actores & Nuevos actores & Nuevos actores & & \\
\hline Transparencia & & $\begin{array}{l}\text { Mecanismos de } \\
\text { acceso abierto a } \\
\text { la información }\end{array}$ & $\begin{array}{l}\text { Mecanismos de } \\
\text { acceso abierto a } \\
\text { la información }\end{array}$ & $\begin{array}{l}\text { Flujos de } \\
\text { información } \\
\text { horizontal entre } \\
\text { entidades y } \\
\text { entre estas y las } \\
\text { autoridades }\end{array}$ & $\begin{array}{l}\text { Más } \\
\text { información } \\
\text { actividad } \\
\text { del Ayto. }\end{array}$ & $\begin{array}{l}\text { Más } \\
\text { información } \\
\text { actividad } \\
\text { del Ayto. }\end{array}$ \\
\hline Autoridad & Inicial & $\begin{array}{l}\text { Reconocimiento } \\
\text { posterior de } \\
\text { las propuestas } \\
\text { realizadas en } \\
\text { el marco de } \\
\text { las asambleas } \\
\text { (mapa de } \\
\text { necesidades) }\end{array}$ & Inicial & $\begin{array}{l}\text { Iniciativa, } \\
\text { implementación } \\
\text { y evaluación de } \\
\text { la política de } \\
\text { voluntariado } \\
\text { (coproducción) }\end{array}$ & & \\
\hline Obstáculos & $\begin{array}{l}\text { Conflicto } \\
\text { con } \\
\text { entidades y } \\
\text { relaciones } \\
\text { bilaterales } \\
\text { paralelas }\end{array}$ & $\begin{array}{l}\text { Prácticas de } \\
\text { auto-selección } \\
\text { y sesgos } \\
\text { políticos en la } \\
\text { participación }\end{array}$ & $\begin{array}{l}\text { Sesgos } \\
\text { políticos en la } \\
\text { implementación }\end{array}$ & $\begin{array}{l}\text { Reducción } \\
\text { a política de } \\
\text { voluntariado }\end{array}$ & $\begin{array}{l}\text { Carácter } \\
\text { informativo, } \\
\text { otros } \\
\text { canales de } \\
\text { influencia }\end{array}$ & $\begin{array}{l}\text { Carácter } \\
\text { informativo, } \\
\text { otros } \\
\text { canales de } \\
\text { influencia }\end{array}$ \\
\hline
\end{tabular}

Fuente: elaboración propia.

Como muestra la Tabla 3, los casos estudiados en experiencias de tipo intermedio - aun no siendo los más exitosos - dan lugar a procesos de cambio en las relaciones y en el contexto político local. Esos cambios son modestos y, en la mayoría de los casos, no llegan a consolidarse cuando los procesos participativos finalizan. No obstante, instituciones como los presupuestos participativos promueven una mayor pluralidad en las redes de políticas, una mayor transparencia en la gestión de la información, fomentan la influencia política y la autoridad de los participantes y grupos asociativos. Estos procesos son incipientes y, en todos los casos, se encuentran con la resistencia de actores políticos dominantes en la red de políticas locales (ya sean las propias autoridades o entidades asociativas tradicionalmente más poderosas). En contraste, los consejos consultivos estudiados promueven cambios más limitados: aumentan la sociabilidad entre los actores, el flujo de información vertical y, sólo en un caso, transforman el flujo de información en un sentido más multilateral y coopera- 
tivo. Sólo en ese caso de un consejo sectorial observábamos también transformaciones más ambiciosas en términos de autoridad de las entidades asociativas.

Podríamos concluir que el tipo de instrumento participativo y la resistencia que oponen los actores dominantes constituyen dos elementos clave para entender la capacidad transformativa de estos instrumentos participativos. La inercia de las relaciones previas supone un obstáculo a considerar a la hora de evaluar la efectividad de las instituciones de participación ciudadana.

\section{Conclusiones}

Quizás los procesos participativos sean algo menos singulares de lo que hemos empezado suponiendo al iniciar este texto. Seguramente habrá muchos conductores que serán felices con su coche si este es capaz de producir la admiración y envidia de sus vecinos. Y muchos seguidores de la selección de balonmano sentirán que ese entrenador es el que quieren si les hace sentirse orgulloso de su equipo, aunque siga sin ganar finales. Incluso habrá personas que valoren a la escuela pública en función de si mantiene a sus hijos controlados durante un amplio horario o que considerarán que el hospital funciona mejor en función de cuantos titulares positivos genere en los medios de comunicación.

Puede que en casi todos los casos haya cierta ambigüedad sobre que significa ser efectivo o simplemente criterios encontrados sobre en que consiste esa efectividad para cada una de nosotras. Pero es muy probable que los procesos participativos sufran algo más aún esa dificultad dada la ausencia de criterios de evaluación ampliamente compartidos y dada la muy diversa gama de objetivos explícitos o implícitos que estos pueden tener. En todo caso, hemos empezado este texto apuntando los grandes rasgos de ese debate, principalmente para que el lector pueda orientarse si quiere encontrar más información al respecto.

En el grueso del artículo nos hemos centrado en uno de los criterios desde los que podríamos abordar esa eficacia, las consecuencias sustantivas de esos procesos. Primero hemos abordado la pregunta de hasta qué punto estos procesos tienen un impacto en las políticas adoptadas por las administraciones públicas y hemos visto como una investigación reciente, de entre las más ambiciosas y diversas realizadas hasta ahora en ningún país sobre el tema, apunta a que el grado de traslado de estas propuestas a políticas públicas es algo mayor de lo que parecía apuntar buena parte de las investigaciones previas. Tam- 
bién es cierto que esta aparente buena noticia queda ensombrecida por varios matices. En primer lugar, por el hecho de que la mayor parte de estas propuestas son muy modestas y poco ambiciosas en sus objetivos, lo que facilita esa implementación, pero quizás dificulta que se lleguen a colmar las amplias expectativas con las que estos procesos son presentados. Aunque aquí no hemos entrado en los detalles de cuáles son los factores que explican que unas propuestas tiendan a implementarse más que otras, sí hemos visto que existe un amplio margen para que los políticos suelan elegir solo parte de las mismas para su ejecución. A esa realidad formada por tres grandes tercios que hemos dibujado anteriormente (un tercio de propuestas totalmente implementadas sin cambios, un tercio de implementación parcial y otro tercio de rechazos) hay que añadirle una limitada tradición de dar explicaciones: aunque en casi dos tercios de los casos la decisión se ha desviado de la propuesta inicial (no implementándose o haciendo de manera parcial), en menos de la mitad de esas desviaciones se ha dado algún tipo de explicación pública de porque esto ha sido así. Será necesario seguir investigando sobre el tema para ver hasta qué punto estas tendencias se producen también en otros contextos y realidades y seguir reflexionando con los actores implicados para ver qué cambios pueden adoptarse en las prácticas participativas para evitar que estas generen frustración.

Ese es precisamente el otro gran tema que hemos abordado aquí. Los procesos participativos pueden suponer cambios en las políticas, pero también tienen efectos sobre el contexto político, en los actores políticos y en sus modos de interacción. A partir de un estudio de seis casos bastante comunes de consejos consultivos y presupuestos participativos hemos observado varias tendencias a pequeños cambios en positivo que se dan tanto en la red de actores sociales presentes, como en sus relaciones con las administraciones públicas. Los matices en negativo que es necesario introducir en este caso vienen de dos limitaciones. En primer lugar, estos cambios no se dan en todos los casos, con cierta tendencia a que se den con mayor frecuencia en presupuestos participativos e incluso sólo en los mejores de entre estos. En segundo lugar, estos cambios no son siempre en positivo. Las situaciones de conflicto y frustración de expectativas son también parte de la realidad.

Ser conscientes de estas limitaciones de los procesos participativos no tiene por qué llevar a la total negación de sus potenciales, pero sí a iniciar un diálogo constructivo sobre qué podemos y que no podemos alcanzar con los mismos, que incluya expectativas realistas sobre sus alcances y que incorpore también sus limitaciones y sus riesgos en ese debate. 


\section{Referencias}

ALARCÓN, P., \& FONT, J. (2014). Where Are the Boundaries of Deliberation and Participation? ATransatlantic Debate. Journal of Public Deliberation, 10(2).

BAIOCCHI, G., HELLER, P., \& SILVA, M. K. (2008). Making space for civil society: institutional reforms and local democracy in Brazil. Social Forces, 86(3), 911-936.

BAIOCCHI, G., HELLER, P., SILVA, M. K., \& SILVA, M. (2011). Bootstrapping democracy: Transforming local governance and civil society in Brazil. Stanford University Press.

BAIOCCHI, G. (2005). Militants and citizens. The politics of participatory democracy in Porto Alegre. Stanford: Stanford University Press.

BHERER, L., FERNÁNDEZ-MARTÍNEZ, J., GARCÍA-ESPÍN, P. y JIMÉNEZ, M. (2006). The Promise for Democratic Deepening: the Effects of Participatory Processes in the Interaction between Civil Society and Local Governments. Journal of Civil Society (en curso de publicación).

BOULDING, C. yWAMPLER, B. (2009). "Voice, votes and resources: evaluating the effect of participatory democracy on well-being", World Development, 38 (1): 125-135.

CARRERAS, F y VALLÉS, J.M. (1977). Las elecciones: introducción a los sistemas electorales, Barcelona: Blume.

FAGOTTO, E., \& FUNG, A. (2006). Empowered participation in urban governance: the Minneapolis neighborhood revitalization program. International Journal of Urban and Regional Research, 30(3), 638-655.

FAGOTTO, E., \& FUNG, A. (2009). Sustaining public engagement: Embedded deliberation in local communities. East Hartford, CT: Everyday Democracy \& Kettering Foundation.

FEREJOHN, J. A. (1974). Pork Barrel Politics: Rivers and Harbors Legislation, 1947-1968 (Stanford, CA: Stanford University Press).

FIORINA, M. (1999). "Extreme Voices:The Dark Side of Civic Engagement», in Skocpol y Fiorina (eds): Civic engagement in American democracy, Brookings institution, $\mathrm{p}$ 395-426.

FONT, J y MEDINA, L (2001). "Consejos ciudadanos en España: ¿un despilfarro europeo?», en Font, J. (ed): Ciudadanos y decisiones públicas, Barcelona: Ariel, p 153-174.

FONT, J. y BLANCO, I. (2006). Polis, la ciudad participativa. Papers de participació ciutada, 9, Diputació de Barcelona.

FONT, J., DEL AMO, S. P., \& Smith, G. (2016). Tracing the Impact of Proposals from Participatory Processes: Methodological Challenges and Substantive Lessons. Journal of Public Deliberation, 12(1).

FUNES, M. J. (2016). ¿Facilitan los proyectos institucionales de participación la capacitación política? Política y Sociedad, 53(1), 55-77. 
FUNES, M. J., TALPIN, J. \& RULL, M. (2014). The cultural consequences of engagement in democratic processes. In J. Font, D. Della Porta, \&Y. Sintomer. Participatory democracy in Southern Europe: causes, characteristics and consequences. London: Rowman \& Littlefield International.

FUNG, A. (2006). "Varieties of participation in complex governance», Public Administration Review, December, p 66-75.

FUNG, A. (2009). Empowered participation: reinventing urban democracy. Princeton University Press.

GANUZA, E y FRANCÉS, F. (2012). El círculo virtuoso de la democracia: los presupuestos participativos a debate, Madrid: CIS.

GARCÍA ESPIN, P., \& JIMÉNEZ SANCHEZ, M. (2015). Los efectos de los procesos participativos en la sociedad civil. La opinión de los expertos. Documento de trabajo IESA/CSIC.

GÓMEZ, B y FONT, J. (2015). Cómo votamos en los referéndums, Madrid: La Catarata-Fundación Alternativas.

GOODIN, ROBERT E. y DRYZEK, JOHN S. (2006). «Deliberative Impact:The Macro-political Uptake of Mini-Publics», Politics and Society, 34: 1-26.

JORBA, L. y ANDUIZA, E. (2009) "Por qué y cómo evaluar la participación», en M. Parés, coord., Participación y calidad democrática. Evaluando las nuevas formas de democracia participativa, Barcelona, Editorial Ariel.

JOSS, S. (1998). «Danish Consensus Conferences as a Model of Participatory Technology Assessment: An Impact Study of Consensus Conferences on Danish Parliament and Danish Public Debate», Science and Public Policy, 25: 2-22.

LOWNDES, V.; PRATCHETT, L y STOKER, G. (2001). "Trends in Public Participation: Part 2 - "Citizens" Perspectives", Public Administration, 79: 445-455.

MAZEAUD, A., SA VILAS, M. y BERTHOMÉ, G. (2012). «Penser les effets de la participation sur l'action publique à partir de ses impensés», Participations, $1: 6-29$.

MORRELL, M. E. (1999). Citizen's evaluations of participatory democratic procedures: Normative theory meets empirical science. Political Research Quarterly, 52(2), 293-322.

PAPADOPOULOS, Y. y WARIN, P. (2007), "Are innovative, participatory and deliberative procedures in policy making democratic and effective?». European Journal of Political Research, 46: 445-472.

PINCOCK, H. (2012). Does deliberation make better citizens. Democracy in motion: Evaluating the practice and impact of deliberative civic engagement, 135-162.

SINTOMER,Y., HERZBERG, C. y RÖCKE, A. (2008). «Participatory Budgeting in Europe: Potentials and Challenges", International Journal of Urban and Regional Research, 32 (1): 164-178. 
SMITH, G. (2009): Democratic innovations, Cambridge: Cambridge University Press.

TALPIN, J. (2012). Schools of democracy: How ordinary citizens (sometimes) become competent in participatory budgeting institutions. ECPR Press.

TALPIN, J. (2012b). What can ethnography bring to the study of deliberative democracy? Evidence from a study on the impact of participation on actors. Revista Internacional de Sociología, 70, 143-163.

TATENHOVE, J., EDELENBOS, J. y KLOK, P. (2010). "Power and interactive policy-making: a comparative study of power and influence in 8 interactive projects in the Netherlands", Public Administration, 88 (3): 609-626.

VERBA, S. (1996). "The Citizen as Respondent: Sample Surveys and American Democracy Presidential Address, American Political Science Association, 1995", The American Political Science Review, Vol. 90, pp. 1-7

WAMPLER, B. (2004). Expanding accountability through participatory institutions: Mayors, citizens, and budgeting in three Brazilian municipalities. Latin American Politics and Society, 46(2), 73-99.

WAMPLER, B. (2007). Can Participatory Institutions Promote Pluralism? Mobilizing. Social Science Quarterly, 41(4), 57-78. 
LABURPENA: Parte hartzeko prozesuak hainbat helbururekin jartzen dira martxan, eta, horrenbestez, horien eraginkortasuna aztertzea ez da erraza izaten. Prozesu horiek helburu zehatzak izaten dituzte; esaterako, politika publikoak prestatzea (policies), edo tokiko testuinguru politikoetan aldaketa positiboak sustatzea (polities). Artikulu honetan bi dimentsio horiek ditugu hizpide: Parte hartzeko prozesuak politika publiko berriak zein baldintzatan eragiten dituzten aztertu dugu, eta aldaketa esanguratsuak zein kasutan eragin ditzaketen tokiko testuinguru politikoetan. 2012. eta 2015. urteen artean egindako ikerketa-proiektu baten bitartez, parte hartzeko prozesu ugari aztertu ditugu, eta tokiko eragile publikoen arteko harreman berriak sortu edo politika berriak ekoizteko orduan dituzten mugak eta aukerak hausnarketarako gai izan ditugu.

GAKO HITZAK: Tokiko parte-hartzea. Politika publikoak. Kontsulta-batzordeak. Aurrekontu parte-hartzaileak.

RESUMEN: Los procesos participativos se ponen en marcha con objetivos de distinta índole, por lo que no resulta sencillo abordar el análisis de su efectividad. Estos procesos incorporan objetivos específicos como la producción de políticas públicas (policies), o la promoción de cambios positivos en los contextos políticos locales (polities). En este artículo, nos concentramos en esas dos dimensiones. Nos planteamos en qué condiciones los procesos participativos generan nuevas políticas públicas y en qué casos producen cambios significativos en los contextos políticos locales. A través de un proyecto de investigación realizado entre 2012 y 2015, analizamos una diversidad de procesos participativos y reflexionamos sobre sus límites y oportunidades a la hora de producir nuevas políticas o de crear nuevas relaciones entre los actores públicos locales.

PALABRAS CLAVE: Participación local. Políticas públicas. Consejos consultivos. Prepuestos participativos.

ABSTRACT: Participative processes are implemented with different objectives so it is not easy to address the analysis of their effectiveness. Theses processes have specific objectives such as production of public policies or the promotion of positive changes within local polities. In this article, we focus on those two dimensions. We consider the conditions participative processes cause in local policies and the cases where significant changes over local polities are produced. By means of a research project developed between 2012 and 2015, we analyze the diversity of participative processes and we reflect on their limits and opportunities in order to produce new policies or create new relationships between local public actors.

KEYWORDS: Local participation. Public policies. Consultive councils. Participative budgets. 